\title{
The arithmetic of the coefficients of half-integral weight Eisenstein series
}

\author{
by
}

\begin{abstract}
Antal Balog (Budapest), William J. McGraw (Madison, WI) and Ken Ono (Madison, WI)
\end{abstract}

1. Introduction and statement of results. If $H(-n)$ denotes the Hurwitz-Kronecker class number of positive definite binary quadratic forms with discriminant $-n$, then Zagier's weight $3 / 2$ Eisenstein series is given by ( $q=e^{2 \pi i z}$ throughout)

$$
H_{3 / 2}(z)=-\frac{1}{12}+\sum_{n=1}^{\infty} H(1, n) q^{n}=-\frac{1}{12}+\sum_{0<n \equiv 0,3(\bmod 4)} H(-n) q^{n} .
$$

This series fits into Cohen's general theory of weight $k+1 / 2$ Eisenstein series

$$
H_{k+1 / 2}(z)=\sum_{n=0}^{\infty} H(k, n) q^{n},
$$

where $H(k, n)=H_{1}(k, n)$ is defined in (1.4). If $k \geq 2$, then $H_{k+1 / 2}(z)$ is a weight $k+1 / 2$ modular form on the congruence subgroup $\Gamma_{0}(4)$ (see [3, Th. 3.1]).

If $D \equiv 0,1(\bmod 4)$, and $\chi_{D}(\bullet)=\left(\frac{D}{\bullet}\right)$ denotes the usual Kronecker character, then it is a classical fact (see $[1$, Ch. $5, \S 4]$ ) that

$$
H(1,-D)=H(D)=\sum_{a=0}^{|D|-1} \chi_{D}(a) a,
$$

when $D<-4$ is a fundamental discriminant.

We generalize this classical result in a variety of ways. See, for example, Corollary 1.2. Moreover, we obtain an elegant uniform description of the

2000 Mathematics Subject Classification: Primary 11F11.

The authors thank the National Science Foundation for their generous support. The second and third authors thank the Number Theory Foundation for their generous support, and the third author is grateful for the support of the Alfred P. Sloan, David and Lucile Packard, and H. I. Romnes Fellowships. 
$H(k, n)$ in terms of values of the Bernoulli polynomials $B_{k}(x)$, weighted against the $\bmod n$ representation numbers of sums of squares.

To state our result, if $\chi$ is a Dirichlet character, we let $L(s, \chi)=$ $\sum \chi(n) n^{-s}$ be its $L$-series. For positive integers $r, n$ and $k$ with $r$ odd, let $D:=(-1)^{k} n$ and set

$$
\begin{aligned}
& h_{r}(k, n) \\
&= \begin{cases}\frac{(-1)^{[k / 2]} \chi_{8}(r)(k-1) ! n^{k-r / 2} L\left(k, \chi_{D}\right)}{2^{k-(r+1) / 2} \pi^{k}} & \text { if }(-1)^{k} n \equiv 0(\bmod 4), \\
\frac{(-1)^{[k / 2]}(k-1) ! n^{k-r / 2} L\left(k, \chi_{D}\right)}{2^{k-1} \pi^{k}} & \text { if }(-1)^{k} n \equiv 1(\bmod 4), \\
0 & \text { if }(-1)^{k} n \equiv 2,3(\bmod 4) .\end{cases}
\end{aligned}
$$

Define also

$$
H_{r}(k, n)= \begin{cases}\sum_{d^{2} \mid n} h_{r}\left(k, n / d^{2}\right) & \text { if }(-1)^{k} n \equiv 0,1(\bmod 4), \\ \zeta(1-2 k) & \text { if } n=0, \\ 0 & \text { otherwise. }\end{cases}
$$

Note that $h_{r}(k, n) \ll n^{k-r / 2} \log (2 n)$ (where the $\log (2 n)$ is necessary only for $k=1)$. In particular,

$$
H_{r}(k, n) \ll n^{\max (k-r / 2,0)+\varepsilon} .
$$

Next, we define, for positive integers $r$ and $n$,

$$
R_{r}(a, n):=\#\left\{\left(\nu_{1}, \ldots, \nu_{r}\right) \in(\mathbb{Z} / n \mathbb{Z})^{r}: \nu_{1}^{2}+\ldots+\nu_{r}^{2} \equiv a(\bmod n)\right\} .
$$

If $k$ is a positive integer, then let $B_{k}$ be the $k$ th Bernoulli number and let $B_{k}(x)$ denote the usual $k$ th Bernoulli function with the convention that

$$
B_{1}(x)= \begin{cases}x-1 / 2 & \text { if } x \neq 0 \\ 0 & \text { if } x=0 .\end{cases}
$$

Finally, define the two formal Dirichlet series $H_{r, k}(s)$ and $F_{r, k}(s)$ by

$$
\begin{aligned}
& H_{r, k}(s)=\sum_{n=1}^{\infty} \frac{H_{r}(k, n)}{n^{s}} \\
& F_{r, k}(s)=-\frac{\chi_{(-1)^{k}}(r)}{k} \sum_{n=1}^{\infty} \frac{\sum_{a=0}^{n-1} R_{r}(a, n) B_{k}(a / n)}{n^{s}} .
\end{aligned}
$$

By (1.5), $H_{r, k}(s)$ converges for $\operatorname{Re}(s)>\max (1, k+1-r / 2)$. For $F_{r, k}(s)$, we note that $B_{k}(x)$ is bounded in the interval $[0,1]$ and $\sum R_{r}(a, n)=n^{r}$. This implies that $F_{r, k}(s)$ converges for $\operatorname{Re}(s)>r+1$. As a consequence of the following theorem, we can extend the region of convergence for $F_{r, k}(s)$ to $\operatorname{Re}(s)>\max (r / 2+1, r-k+1)$.

By modifying an argument of J. Bruinier (see [2, Th. 11]) in the case where $k=1$, for positive odd integers $r$, we obtain the following descrip- 
tion of $H_{r, k}(s)$ as a formal product of $F_{r, k}(s)$ and a simple quotient of the Riemann zeta function $\zeta(s)$. In particular, if $r=1$, we obtain a convenient description of the Mellin transform of Cohen's half-integral weight Eisenstein series.

THEOREM 1.1. If $r$ is a positive odd integer and $k \geq 1$, then

$$
H_{r, k}(s)=\frac{\zeta(2 s)}{\zeta(s)} F_{r, k}(s-k+r) .
$$

As is well known, $\zeta(2 s) / \zeta(s)=\sum_{n=1}^{\infty} \lambda(n) n^{-s}$, where $\lambda$ is the Liouville function, the totally multiplicative function on positive integers defined on primes $p$ by $\lambda(p)=-1$. Then Theorem 1.1 gives an immediate formula for $H_{r}(k, n)$.

Corollary 1.2. For $r, n, k \in \mathbb{Z}$ with $r$ odd,

$$
H_{r}(k, n)=-\frac{\chi_{(-1)^{k}}(r)}{k} \sum_{d \mid n} \lambda(n / d) d^{k-r} \sum_{a=0}^{d-1} R_{r}(a, d) B_{k}(a / d) .
$$

We cannot resist interpreting the $r=1$ case of Theorem 1.1 in terms of the logarithmic derivatives of the infinite products

$$
F_{k}(q)=\prod_{n=1}^{\infty}\left(1-q^{n}\right)^{H(k, n) / n}
$$

Corollary 1.3. If $k$ is a positive integer, then

$$
\frac{q \frac{d}{d q}\left(F_{k}(q)\right)}{F_{k}(q)}+\sum_{\substack{a, b \geq 1 \\ c \geq 2 \\ b \text { square-free }}} H(k, a) q^{a b c^{2}}=\frac{1}{k} \sum_{n=1}^{\infty} \sum_{a=0}^{n-1} R_{1}(a, n) B_{k}(a / n) n^{k-1} q^{n} .
$$

Acknowledgments. The authors thank the referee, whose contributions improved this paper.

2. Proofs. We begin with the following elementary lemmas. The first lemma is standard and can be found in [4, p. 522].

Lemma 2.1. The Fourier expansion of $B_{k}(x)$ is given by

$$
B_{k}(x)=\frac{-k !}{(2 \pi i)^{k}} \sum_{n \in \mathbb{Z}}^{\prime} \frac{e(n x)}{n^{k}},
$$

where $\sum^{\prime}$ indicates that we are summing over all non-zero integers, and $e(x)=e^{2 \pi i x}$.

The proof of the following lemma is a straightforward analogue of the proof of Möbius inversion and will be left to the reader. 
Lemma 2.2. Let $f: \mathbb{Z}^{+} \rightarrow \mathbb{C}$. Define $F, G: \mathbb{Z}^{+} \rightarrow \mathbb{C}$ by

$$
F(N)=\sum_{d \mid N} f(N / d), \quad G(N)=\sum_{d^{2} \mid N} f\left(N / d^{2}\right) .
$$

Then

$$
\begin{aligned}
\text { (i) } F(N) & =\sum_{\substack{a \mid N \\
N / \text { s square-free }}} G(a), \\
\text { (ii) } \quad G(N) & =\sum_{a \mid N} \lambda(N / a) F(a) .
\end{aligned}
$$

Fixing notation, we define, for integers $m$ and $a$,

$$
G(m, a)=\sum_{\nu \in \mathbb{Z} / a \mathbb{Z}} e\left(m \nu^{2}\right)
$$

In the following lemma, we recall some basic facts on the Gauss sums in (2.4). For their proofs, we refer the reader to [5, IV.3].

Lemma 2.3. Suppose that $a, b$ and $c$ are integers with $b, c \geq 1$.

(i) If $c \mid(a, b)$, then $G(a, b)=c \cdot G(a / c, b / c)$.

(ii) If $(b, c)=1$ and $(a, b c)=1$, then $G(a, b c)=G(a b, c) G(a c, b)$.

(iii) If $b$ is odd and $(a, b)=1$, then $G(a, b)=\left(\frac{a}{b}\right) G(1, b)$.

(iv) If $a$ is odd, then $G\left(a, 2^{s}\right)=\left(\frac{-2^{s}}{a}\right) \varepsilon((a-1) / 2) G\left(1,2^{s}\right)$.

$$
\text { (v) } G(1, b)= \begin{cases}(1+i) \sqrt{b} & \text { if } b \equiv 0(\bmod 4), \\ \sqrt{b} & \text { if } b \equiv 1(\bmod 4), \\ 0 & \text { if } b \equiv 2(\bmod 4), \\ i \sqrt{b} & \text { if } b \equiv 3(\bmod 4)\end{cases}
$$

Here, $\varepsilon(b)=1$ if $b$ is even and $\varepsilon(b)=i$ if $b$ is odd.

For positive integers $k$ and $r$, let

$$
\mathfrak{g}_{k, r}(m, a)= \begin{cases}\operatorname{Re}\left(G(m, a)^{r}\right) & \text { if } k \in 2 \mathbb{Z} \\ \operatorname{Im}\left(G(m, a)^{r}\right) & \text { if } k \in 2 \mathbb{Z}+1\end{cases}
$$

Then we see immediately from the definition and (i) above that, for $b \mid(m, a)$,

$$
\mathfrak{g}_{k, r}(m, a)=b^{r} \mathfrak{g}_{k, r}(m / b, a / b) .
$$

Then we have the following proposition.

Proposition 2.4. For integers $m$ and a such that $(m, a)=1$,

$$
\begin{aligned}
& \mathfrak{g}_{k, r}(m, a) \\
& =a^{r / 2}\left(\frac{(-1)^{k} a}{m}\right) \cdot \begin{cases}2^{(r-1) / 2} \chi_{(-1)^{k}}(r) & \text { if }(-1)^{k} a \equiv 0(\bmod 4), \\
\chi_{(-1)^{k}}(r) & \text { if }(-1)^{k} a \equiv 1(\bmod 4), \\
0 & \text { if }(-1)^{k} a \equiv 2,3(\bmod 4) .\end{cases}
\end{aligned}
$$


Proof. If $(-1)^{k} a \equiv 1(\bmod 4)$, then $G(m, a)^{r}=\left(\frac{m}{a}\right)^{r} \varepsilon(k)^{r} a^{r / 2}$. Therefore,

$$
\mathfrak{g}_{k, r}(m, a)=a^{r / 2}\left(\frac{m}{a}\right) \varepsilon(k)^{r-1}=a^{r / 2}\left(\frac{m}{a}\right) \chi_{(-1)^{k}}(r) .
$$

Clearly, by Lemma 2.3 , if $(-1)^{k} a \equiv 2(\bmod 4)$, then $\mathfrak{g}_{k, r}(m, a)=0$. If $(-1)^{k} a \equiv 3(\bmod 4)$, then $G(m, a)^{r}=\left(\frac{m}{a}\right)^{r} \varepsilon(k-1)^{r} a^{r / 2}$ and $\mathfrak{g}_{k, r}(m, a)=0$.

Therefore, the only case left is $(-1)^{k} a \equiv 0(\bmod 4)$. Suppose $a=2^{s} a_{0}$ with $a_{0}$ odd; then, by Lemma 2.3 , we see that

$$
G(m, a)=\left(\frac{-m}{a_{0}}\right)\left(\frac{-2^{s}}{m}\right) \varepsilon\left(\frac{m a_{0}-1}{2}\right) G\left(1,2^{s}\right) G\left(1, a_{0}\right) .
$$

Therefore, if $a_{0} \equiv 1(\bmod 4)$, then

$$
\begin{aligned}
G(m, a) & =\left(\frac{-2^{s} a_{0}}{m}\right) \varepsilon\left(\frac{m-1}{2}\right)(1+i) 2^{s / 2} \sqrt{a_{0}} \\
& =\left(\frac{-a}{m}\right) \varepsilon\left(\frac{m-1}{2}\right) \sqrt{a}(1+i),
\end{aligned}
$$

and if $a_{0} \equiv 3(\bmod 4)$, then

$$
\begin{aligned}
G(m, a) & =-\left(\frac{-a_{0}}{m}\right)\left(\frac{-2^{s}}{m}\right) \varepsilon\left(\frac{m+1}{2}\right) i \sqrt{a_{0}}(1+i) 2^{s / 2} \\
& =-\left(\frac{a}{m}\right) \varepsilon\left(\frac{m+1}{2}\right) i \sqrt{a}(1+i) \\
& =\left(\frac{-a}{m}\right) \varepsilon\left(\frac{m-1}{2}\right) \sqrt{a}(1+i) .
\end{aligned}
$$

Then we see that

$$
G(m, a)^{r}=2^{(r-1) / 2} a^{r / 2}\left(\frac{-a}{m}\right) \varepsilon\left(\frac{m-1}{2}\right)^{r} i^{(r-1) / 2} .
$$

After a lengthy verification, we obtain

$$
\mathfrak{g}_{k, r}(m, a)=\left(\frac{(-1)^{k} a}{m}\right) 2^{(r-1) / 2} a^{r / 2} \chi_{(-1)^{k} 8}(r),
$$

as desired.

The following proposition provides the foundation for Theorem 1.1.

Proposition 2.5. For $r, k, n \in \mathbb{Z}^{+}$with $r$ odd,

$$
\sum_{a=0}^{n-1} R_{r}(a, n) B_{k}(a / n)=-\frac{\chi_{(-1)^{k}}(r) k}{n^{k-r}} \sum_{d \mid n} h_{r}(k, d) .
$$


Proof. Let $N$ be a positive integer; then by Lemma 2.1,

$$
\begin{aligned}
\sum_{a=0}^{N-1} R_{r}(a, N) B_{k}(a / N) & \sum_{\nu_{1}, \ldots, \nu_{r}(\bmod N)} B_{k}\left(\frac{\nu_{1}^{2}}{N}+\ldots+\frac{\nu_{r}^{2}}{N}\right) \\
= & \frac{-k !}{(2 \pi i)^{k}} \sum_{n \in \mathbb{Z}}^{\prime} n^{-k} \sum_{\nu_{1}, \ldots, \nu_{r}(\bmod N)} e\left(n\left(\frac{\nu_{1}^{2}}{N}+\ldots+\frac{\nu_{r}^{2}}{N}\right)\right) \\
= & \frac{-k !}{(2 \pi i)^{k}} \sum_{n \in \mathbb{Z}}^{\prime} n^{-k} G(n, N)^{r} \\
= & \frac{-k !}{(2 \pi i)^{k}} \sum_{n \in \mathbb{Z}}^{\prime} n^{-k}\left(\operatorname{Re}\left(G(n, N)^{r}\right)+i \operatorname{Im}\left(G(n, N)^{r}\right)\right) \\
= & \frac{-2 \varepsilon(k) k !}{(2 \pi i)^{k}} \sum_{n \geq 1} n^{-k} \mathfrak{g}_{k, r}(n, N) .
\end{aligned}
$$

Reindexing this sum on the divisors of $N$, we find

$$
\begin{aligned}
\sum_{a=0}^{N-1} R_{r}(a, N) & B_{k}(a / N) \\
= & \frac{-2 \varepsilon(k) k !}{(2 \pi i)^{k}} \sum_{a \mid N} \sum_{\substack{n \geq 1 \\
(n, N)=N / a}} n^{-k} \mathfrak{g}_{k, r}(n, N) \\
= & \frac{-2 \varepsilon(k) k !}{(2 \pi i)^{k}} N^{r-k} \sum_{a \mid N} a^{k-r} \sum_{\substack{m \geq 1 \\
(m, a)=1}} m^{-k} \mathfrak{g}_{k, r}(m, a) .
\end{aligned}
$$

Since $\chi_{a}(m)=0$ if $(m, a) \neq 1$, Proposition 2.4 yields

$$
\begin{aligned}
a^{k-r} \sum_{\substack{m \geq 1 \\
(m, a)=1}} m^{-k} \mathfrak{g}_{k, r}(m, a) & \\
& =2^{(r-1) / 2} \chi_{(-1)^{k} 8}(r) a^{k-r / 2} \sum_{m \geq 1} m^{-k} \chi_{(-1)^{k} a}(m) \\
& =2^{(r-1) / 2} \chi_{(-1)^{k} 8}(r) a^{k-r / 2} L\left(k, \chi_{(-1)^{k} a}\right)
\end{aligned}
$$

if $(-1)^{k} a \equiv 0(\bmod 4)$, and

$$
\begin{aligned}
a^{k-r} \sum_{\substack{m \geq 1 \\
(m, a)=1}} m^{-k} \mathfrak{g}_{k, r}(m, a) & =\chi_{(-1)^{k}}(r) a^{k-r / 2} \sum_{m \geq 1} m^{-k} \chi_{(-1)^{k} a}(m) \\
& =\chi_{(-1)^{k}}(r) a^{k-r / 2} L\left(k, \chi_{(-1)^{k} a}\right)
\end{aligned}
$$


if $(-1)^{k} a \equiv 1(\bmod 4)$. If $(-1)^{k} a \equiv 2,3(\bmod 4)$, the sum is zero. (1.3) yields

$$
\sum_{a=0}^{N-1} R_{r}(a, N) B_{k}(a / N)=-\frac{\chi_{(-1)^{k}}(r) k}{N^{k-r}} \sum_{d \mid N} h_{r}(k, d)
$$

as desired.

We are now prepared to prove the main theorem.

Proof of Theorem 1.1. Combining Proposition 2.5 with Lemma 2.2(ii) yields

$$
H_{r}(k, n)=-\frac{\chi_{(-1)^{k}}(r)}{k} \sum_{d \mid n} \lambda(n / d) d^{k-r} \sum_{a=0}^{d-1} R_{r}(a, d) B_{k}(a / d) .
$$

A straightforward calculation involving formal Dirichlet series shows that, for any series $\sum_{n \geq 1} b(n) n^{-s}$,

$$
\frac{\zeta(2 s)}{\zeta(s)} \sum_{n \geq 1} b(n) n^{-s}=\sum_{n \geq 1}\left(\sum_{d \mid n} \lambda(n / d) b(d)\right) n^{-s} .
$$

Applying this to (2.19), we obtain the theorem.

Proof of Corollary 1.2. This is (2.19) in the proof of Theorem 1.1.

Proof of Corollary 1.3. If we define a formal product

$$
F(q)=\prod_{n=1}^{\infty}\left(1-q^{n}\right)^{c(n)}
$$

then one obtains formally

$$
\frac{q \frac{d}{d q}(F(q))}{F(q)}=-\sum_{n=1}^{\infty}\left(\sum_{d \mid n} c(d) d\right) q^{n} .
$$

Combining Proposition 2.5 with Lemma 2.2(i), we get

$$
-\frac{\chi_{(-1)^{k}}(r)}{n^{r-k} k} \sum_{a=0}^{n-1} R_{r}(a, n) B_{k}(a / n)=\sum_{\substack{d \mid n \\ n / d \text { square-free }}} H_{r}(k, d) .
$$

The result follows from this and (2.21) when $r=1$.

3. Remarks. We remark that these calculations can be carried out in similar settings with similar results. We state some interesting formulas that are obtained when $r$ is even in the above calculations. 
THEOREM 3.1. With notation as above:

(i) If $r \equiv 2(\bmod 4)$ and $k$ is even,

$$
\frac{1}{B_{k}} \sum_{a=0}^{n-1} R_{r}(a, n) B_{k}(a / n)=n^{r-k} \sum_{\substack{d \mid n \\ 2 \nmid d}}\left(\frac{-1}{d}\right) d^{k-r / 2} \prod_{p \mid d}\left(1-\frac{1}{p^{k}}\right) .
$$

(ii) If $r \equiv 2(\bmod 4)$ and $k$ is odd,

$$
\frac{(-1)^{(r-2) / 4}}{2^{r / 2} B_{k}(1 / 4)} \sum_{a=0}^{n-1} R_{r}(a, n) B_{k}(a / n)=n^{r-k} \sum_{\substack{d|n \\ 4| d}} d^{k-r / 2} \prod_{\substack{p \mid d \\ p \neq 2}}\left(1-\frac{\left(\frac{-1}{p}\right)}{p^{k}}\right) \text {. }
$$

(iii) If $r \equiv 0(\bmod 4)$ and $k$ is even,

$$
\begin{gathered}
\frac{1}{B_{k}} \sum_{a=0}^{n-1} R_{r}(a, n) B_{k}(a / n)=n^{r-k} \sum_{d \mid n} d^{k-r / 2} \prod_{p \mid d}\left(1-\frac{1}{p^{k}}\right) \eta(d), \quad \text { where } \\
\eta(d)= \begin{cases}1 & \text { if } 2 \nmid d, \\
0 & \text { if } d \equiv 2(\bmod 4), \\
(-1)^{r / 4} 2^{r / 2} & \text { if } 4 \mid d .\end{cases}
\end{gathered}
$$

(iv) If $r \equiv 0(\bmod 4)$ and $k$ is odd,

$$
\sum_{a=0}^{n-1} R_{r}(a, n) B_{k}(a / n)=0 \text {. }
$$

\section{References}

[1] Z. I. Borevich and I. R. Shafarevich, Number Theory, Academic Press, New York, 1966.

[2] J. Bruinier, On the rank of Picard groups of modular varieties attached to orthogonal groups, Compositio Math. 133 (2002), 49-63.

[3] H. Cohen, Sums involving the values at negative integers of L-functions of quadratic characters, Math. Ann. 217 (1975), 271-285.

[4] K. Knopp, Theory and Application of Infinite Series, 2nd ed., Hafner, New York, 1947.

[5] S. Lang, Algebraic Number Theory, Grad. Texts in Math. 110, Springer, 1986.

Mathematical Institute

Hungarian Academy of Sciences

P.O. Box 127

Budapest 1364, Hungary

E-mail: balog@hexagon.math-inst.hu
Department of Mathematics University of Wisconsin Madison, WI 53706, U.S.A. E-mail: mcgraw@math.wisc.edu ono@math.wisc.edu 